\title{
Estabilización de temperatura en un digestor anaerobio tipo UASB alimentado con residuos bovinos y lodos
}

\section{Temperature stabilization in anaerobic digester U.A.S.B type with waste and sludge powered cattle}

\author{
págs. $69-83$ \\ Grupo de Investigación: Energías Alternativas \\ Línea de investigación: Energías Limpias - Biomasa \\ Andrea Lache Muñoz• Adriana Páez•• Daniela Bernal Vélez •. Daniela Quintero Garzón...•
}

\section{RESUMEN}

Esta investigación evaluó la estabilización de la temperatura en la digestión anaerobia del residuo bovino. El objetivo fue desarrollar una estrategia con base en la temperatura para agilizar la producción de biogás y/o aumentar su calidad en cuanto al porcentaje de metano. Las pruebas experimentales se realizaron en las condiciones climáticas de la Sabana de Bogotá (Colombia). Inicialmente, se establecieron las condiciones de operación del proceso de digestión anaerobio para determinar el parámetro que afecta directamente la producción de biogás. Se construyó un digestor convencional y un digestor nuevo prototipo, al que se le realizó una mejora (el modelo de reactor seleccionado fue el UASB). Esta mejora se determinó a partir del estudio de los parámetros que afectan la digestión anaerobia, donde la temperatura fue el parámetro de mayor incidencia en el proceso y que puede ser controlado externamente. Los resultados permitieron confirmar una mejora en el desempeño del digestor con el sistema de estabilización de temperatura con respecto al digestor convencional.

\begin{abstract}
This paper presents the study of temperature stabilization in the anaerobic co-digestion of cattle manures. The aim was to develop a strategy based on the temperature to speed up the production of biogas and/or increase their quality in the percentage of methane. The trial was conducted in climatic conditions of the savannah of Bogota, Colombia. Initially operating conditions anaerobic digestion process were established to determine the parameter that directly affects the production of biogas $A$ conventional digester and a new prototype digester to which is made an improvement was constructed. This improvement is determined from the study of physical parameters and parameters that affect anaerobic digestion, defining temperature as the parameter that directly affects the process and that can be manipulated externally. The results were satisfactory for the new digester prototype over conventional digester, obtaining a volume of methane $0.018 \mathrm{~m}^{3}$ and $0.015 \mathrm{~m}^{3}$ respectively; likewise biogas quality was higher for the new digester prototype. Therefore the effect of
\end{abstract}

-I.Q. M. Sc. Energías renovables, pilas de combustible de hidrógeno. Docente investigador. Fundación Universidad de América. lache@investigadores.uamerica.edu.co

•- M. Sc. Microbióloga industrial. Docente investigador. Fundación Universidad de América. adriana.paez@investigadores. uamerica edu.co

..• Estudiante coinvestigador. Fundación Universidad de América. daniela.bernal@estudiantes.uamerica.edu.co

...• Estudiante coinvestigador. Fundación Universidad de América. daniela.quintero@estudiantes.uamerica.edu.co 
Se comprobó también que la calidad del biogás fue mayor para el digestor nuevo prototipo. Por lo tanto, se valida la favorabilidad del efecto del control de la temperatura en el rendimiento del proceso de digestión anaerobia para la producción de biogás.

Palabras clave: Bovino, digestión anaerobia, metano. temperature control as benefit anaerobic digestion to biogas production is validated.

Key words: Cattle manure, anaerobic co-digestion, methane.

\section{INTRODUCCIÓN}

Reducir la emisión de gases de efecto invernadero especialmente dióxido de carbono (CO2) ha motivado la implementación de nuevas alternativas para la producción de energía, debido al deterioro medio ambiental que está sufriendo el planeta y en consecuencia a la creciente ocurrencia de fenómenos climáticos adversos. Las fuentes renovables de energía contribuyen a la reducción del uso de fuentes fósiles, cuyas reservas limitadas día a día se agotan, además de que se consideran contaminantes.

La digestión de residuos agrícolas es una alternativa para la generación de energía, que además puede beneficiar a zonas rurales. Por una parte, millones de toneladas de residuos agropecuarios se generan anualmente (FAO, 2008) y por otra, estudios (DANE, 2013) (Borja, Martın, \& Colmenarejo, 2005) revelan la eficiencia, calidad y cantidad de biogás que se puede generar al hacer uso de estos residuos puesto que se encuentran disponibles en todo el mundo. Mediante la digestión, las bacterias acidogénicas convierten la biomasa en ácidos grasos volátiles, que después son consumidos por bacterias metanogénicas y convertidos en gas metano, el cual, posteriormente puede ser transformado en energía eléctrica o térmica. En general, la digestión anaerobia es la tecnología más usada para tratar residuos orgánicos (Upton, 1997), por lo que los esfuerzos han estado centrados en aumentar la eficiencia del proceso y reducir los costos de operación (Pérez, Nieto, \& Fernández Polanco, 2006).

El proyecto consideró el proceso de digestión anaerobia a partir de residuos bovinos en un reactor UASB sometido a condiciones ambientales de la sabana de Bogotá. La experimentación se realizó a escala laboratorio una mejora para la estabilización de la temperatura ya que en investigaciones previas (Bohórquez Guitiérrez Lina, 2004) (Tamrat, Mebeaselassie, \& Amare, 2013), se encuentra que la temperatura es altamente influyente en la eficiencia del proceso de digestión anaerobia y en consecuencia en la cantidad del biogás que se genera.

\section{METODOLOGÍA}

Inicialmente se realizó el montaje de dos digestores anaerobios con un volumen de $60 \mathrm{~L}$, con base en el diseño de un reactor UASB. A uno de los digestores se le adicionó un cambio en la estabilización de la temperatura con el fin de comparar el comportamiento de ambos al final del proceso.

Se establecieron las condiciones de operación del digestor, seguido de la construcción de cada uno de los reactores. Una vez instalados se dio inicio al proceso de digestión, que se evaluó por medio de cuatro variables: temperatura, $\mathrm{pH}, \mathrm{DQO}$ y sólidos. Finalmente se realizó una comparación 
LÍNEA DE INVESTIGACIÓN: ENERGÍAS LIMPIAS - BIOMASA

del tiempo en el inicio de la producción de biogás así como de la cantidad y calidad del mismo en cada uno de los biodigestores.

\section{A. Condiciones de operación}

Las variables de operación que se consideraron fueron temperatura, $\mathrm{pH}$, caudal de entrada del afluente, cantidad de materia orgánica y tiempo de retención, que son parámetros determinantes para el correcto funcionamiento del reactor UASB (Chang F., 2004.).

La temperatura, uno de los factores principales en el proceso de digestión afecta el crecimiento de las bacterias responsables de la producción de biogás (Sánchez E, Travieso L, \& Colmenarejo, 2005). Por ello, se realizó un seguimiento de la temperatura para favorecer que los microorganismos mesofílicos presentes en el reactor realicen el proceso biológico dentro del rango favorable que está entre $25^{\circ} \mathrm{C}$ y $45^{\circ} \mathrm{C}$ (Lobato., 2012.). Las mediciones de temperatura ambiente se tuvieron en cuenta para conocer su variación en el transcurso del día; igualmente, para reconocer la temperatura a la que estaría sometido el digestor, se realizaron mediciones de la temperatura ambiente cuatro veces al día durante todo el proceso. Para monitorear la temperatura interna del digestor se hizo uso de un termómetro bimetálico con precisión de $2^{\circ} \mathrm{C}$.

De igual forma el $\mathrm{pH}$ es otro de los parámetros determinantes para el proceso (Ministerio de energia, 2011), puesto que requiere que se encuentre entre un rango de 6,8 - 7,2 para que las condiciones de crecimiento de los microorganismos sean favorables para el inicio a la digestión (Bautista, 2010). Para la medición de pH se empleó un pH metro OHAUS portátil.

El tiempo de retención hidráulica, indica el tiempo necesario en el que la mezcla debe permanecer en los digestores para que ocurra la fermentación completa y de lugar a la fase metanogénica (Hamed M. \& Wilko K.P, 2004). El TRH se calcula por medio de la ecuación 1, la que está directamente relacionada con la temperatura ambiental a la que se someten los reactores (UPME, Formulación de un programa básico de normalización para aplicaciones de energías alternativas y difusión, Marzo 2003.).

$$
T R H=-51,227 \operatorname{Ln}\left(T^{\circ} \mathrm{C}\right)+206,72
$$

Las fases de la digestión (hidrólisis, acidogénesis, acetogenesis y metanogénesis) se llevaron a cabo en un solo reactor con operación discontinua, es decir, que el reactor se cargó una sola vez; el caudal de entrada del afluente se consideró para los cálculos necesarios del reactor. Con la ecuación 2 se determina el caudal, la cual se tomó del resultado de una investigación bibliográfica en México (Lobato, 2012) donde se empleó este reactor para llevar a cabo la digestión.

$$
V R=T R H^{*} Q * 1440
$$

La cantidad de materia orgánica se calcula a partir del volumen del reactor; inicialmente fue necesario tener en cuenta que el $70 \%$ del volumen del reactor corresponde a la mezcla y el $30 \%$ debe ser para el biogás que se va a generar (Yank L, 2005). Para asegurar que la mezcla degrade la materia orgánica, se consideró una relación 1:3 (estiércol: agua) (Osorio. S, Ciro. V, \& Gonzáles. S, 2007), asegurando el crecimiento de los microorganismos para la digestión. 


\section{B. Construcción de los digestores}

Se seleccionaron tanques herméticos de polietileno de alta densidad para realizar el proceso, tubería PVC, manguera de gas y accesorios necesarios y disponibles comercialmente para asegurar el control y operación de los digestores.

De acuerdo con los parámetros que deben considerarse en los reactores UASB, se calculó el área del reactor, flujo másico, carga hidráulica y velocidad de flujo en la campana teniendo en cuenta las ecuaciones 3 a 6 .

$$
\begin{gathered}
A_{R}=\frac{\pi D^{2}}{4} \\
F=V_{R} * \text { Carga } \\
C_{H}=\frac{Q}{A_{R}} \\
V_{C}=4 * C_{H}
\end{gathered}
$$

\section{Sistema para la estabilización de la temperatura en el digestor}

Para determinar la mejora que se le realizó al digestor se analizaron parámetros que afectan el proceso directamente y parámetros físicos. Teniendo en cuenta la dependencia de cada parámetro, su manipulación y el efecto en el proceso se realizó una matriz de decisión, que permitió conocer la opción más adecuada para realizar la mejora al digestor. De acuerdo con el resultado obtenido se realizó la mejora que consistió en realizar un recubrimiento al digestor, para asegurar una temperatura uniforme en el interior y que los microorganismos mesofílicos presentes realicen el proceso biológico sin variaciones que afecten significativamente el proceso. Adicionalmente, se realizó un recubrimiento del lugar en el que se ubicó el digestor con el fin de proteger el digestor.

El biogás producido durante los procesos de digestión, se almacenó en gasómetros, recipientes inertes, sin riesgos de contaminación microbiológica, sin escapes, ni porosidad y de bajo costo; adaptados a la parte superior de los digestores. 1999).

El cálculo teórico del biogás esperado se calculó por medio de la ecuación 7 (Guevara Vera,

$$
V_{\text {Biogás Total }}=S V^{*} 0,27 * V_{M}
$$

\section{Evaluación de la producción de biogás}

Para dar inicio al proceso fue necesario realizar una prueba de fugas. Posteriormente se elaboraron dos mezclas (estiércol - agua) con el fin de establecer el $\mathrm{pH}$ adecuado, para la primera mezcla se usó estiércol fresco y agua residual, mientras que para la segunda estiércol seco y agua residual. Se procedió a preparar el volumen de carga de los digestores. Adicionalmente, se efectuó una prue- 
LÍNEA DE INVESTIGACIÓN: ENERGÍAS LIMPIAS - BIOMASA

ba al sustrato inicial con el fin de reconocer nutrientes, solidos, carbono orgánico oxidable, relación $\mathrm{C} / \mathrm{N}$ y DQO, y así comparar los resultados al final del proceso. Los parámetros que se analizaron fueron temperatura, $\mathrm{pH}, \mathrm{DQO}$ y sólidos. También, se realizaron dos pruebas microbiológicas, 1) una coloración de Gram para verificar que se la presencia de los microorganismos en el sustrato (los cuales degradan el sustrato); 2) una prueba para evaluar la actividad enzimática y detectar microorganismos proteolíticos, amilolíticos y celulolíticos con el fin de asegurar que los microorganismos presentes degradaran proteínas, celulosa y almidón, permitiendo que la etapa hidrolítica se completara dentro del sistema de digestión anaerobio.

\section{Comparación de la producción de biogás.}

Considerando que el producto de la digestión anaerobia es el biogás, se practicó una cromatografía de gases para conocer su composición y de esta manera hacer una comparación cuantitativa de la cantidad de hidrógeno $\left(\mathrm{H}_{2}\right)$, oxígeno $\left(\mathrm{O}_{2}\right)$ y metano $\left(\mathrm{CH}_{4}\right)$ del biogás en el digestor convencional y en el mejorado. Por último, se planteó un Anova para verificar que los parámetros estudiados y analizados durante el proceso fueran los correctos.

\section{RESULTADOS Y DISCUSIÓN}

De acuerdo a lo anterior, en este apartado se analizan los parámetros medidos durante el proceso para ambos digestores, teniendo en cuenta que estos hacen parte de las condiciones de operación de los digestores.

\section{Temperatura}

El promedio de temperatura ambiente fue de $15,5^{\circ} \mathrm{C}$. Para analizar el comportamiento de la temperatura ambiente e interna en los digestores se registraron lecturas a una misma hora, como se observan en las Figuras 1 y 2.

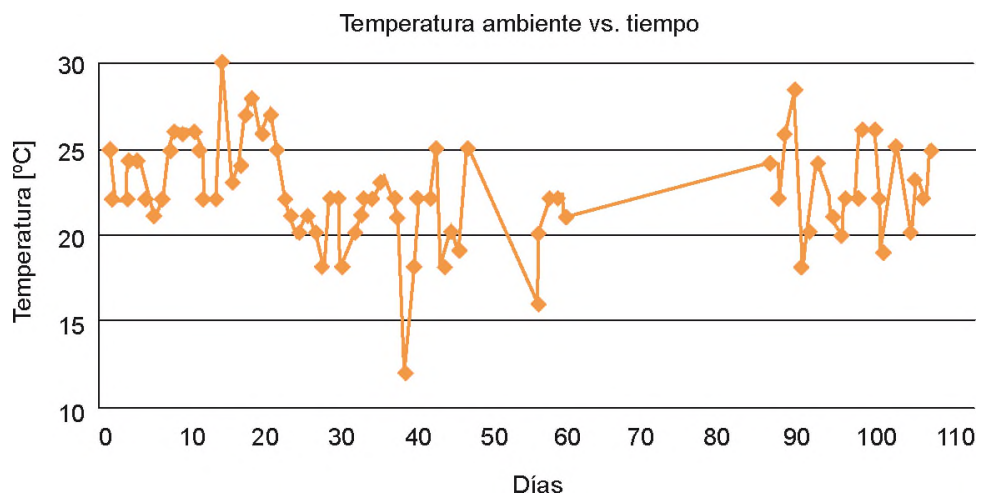

Figura 1. Temperatura ambiente 11:00AM. 
Temperatura vs. tiempo

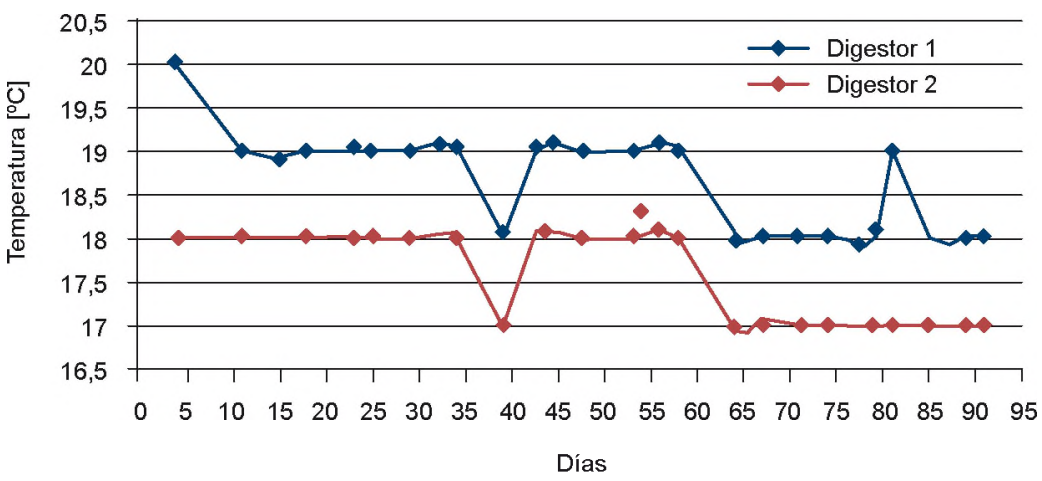

Figura 2. Temperatura interna de los digestores a las 11:00AM.

Con base en las figuras 1 y 2 , y de acuerdo a (Varnero Moreno, 2011), para el adecuado funcionamiento de un digestor se recomienda que el tratamiento anaerobio se diseñe para que opere con variaciones de temperatura que no excedan los $0.6-1.2^{\circ} \mathrm{C} /$ día; esto indica que el digestor con sistema de estabilización de temperatura (digestor 2), cumple lo recomendado, dado que el gradiente de temperatura máximo es de $1^{\circ} \mathrm{C}$ lo que se espera beneficie la producción de biogás (Kalpokas V., 2011).

Ahora bien, al comparar la temperatura ambiente con la temperatura interna del digestor se observa que, a pesar de los cambios, la temperatura fue constante en el digestor, es por esto, que se identificó que el proceso microbiológico (exotérmico) dentro del digestor proporciona la temperatura con la que se lleva a cabo la degradación biológica y así mismo, las propiedades del material de los reactores también favorecen la estabilidad en la temperatura.

\section{$\mathrm{pH}$}

En la etapa inicial del proceso para las mezclas estiércol-agua se reportaron valores de pH más cercanos a la neutralidad para el estiércol fresco (6.8) en la digestión anaerobia; con el cual se procedió a iniciar la digestión.

Según (Yank L, 2005) el pH para la fase hidrolítica corresponde a 7.0-7.2, en la acidogénica se encuentra entre 5.5 y 6.5 , en la metanogénica entre 7.8 y 8.2 y para cultivos mixtos entre 6.8 y 7.4 , siendo el pH neutro el ideal (Bitton, 1994). Otro parámetro directamente relacionado con el pH es la relación $\mathrm{C} / \mathrm{N}$, ya que a mayor contenido de carbono se presenta mayor acidez; por lo tanto un $\mathrm{pH}$ más alto como sucedió con la mezcla de estiércol seco ( $\mathrm{pH}$ 8.6), a diferencia del pH obtenido para el fresco, indica que tiene mayor contenido de nitrógeno, lo que aportó alcalinidad a la mezcla y por consiguiente un valor de $\mathrm{pH}$ cercano a la neutralidad.

Como se puede observar en la figura 3 , el comportamiento de $\mathrm{pH}$ en el digestor 1 y 2 varía conforme van ocurriendo las etapas de la digestió. Cabe precisar que la etapa hidrolítica requiere un $\mathrm{pH}$ más alto con respecto a la etapa acidogénica y que al finalizar se requiere nuevamente un $\mathrm{pH}$ cercano a la neutralidad durante la etapa metanogénica. 
$\mathrm{pH}$ vs. tiempo

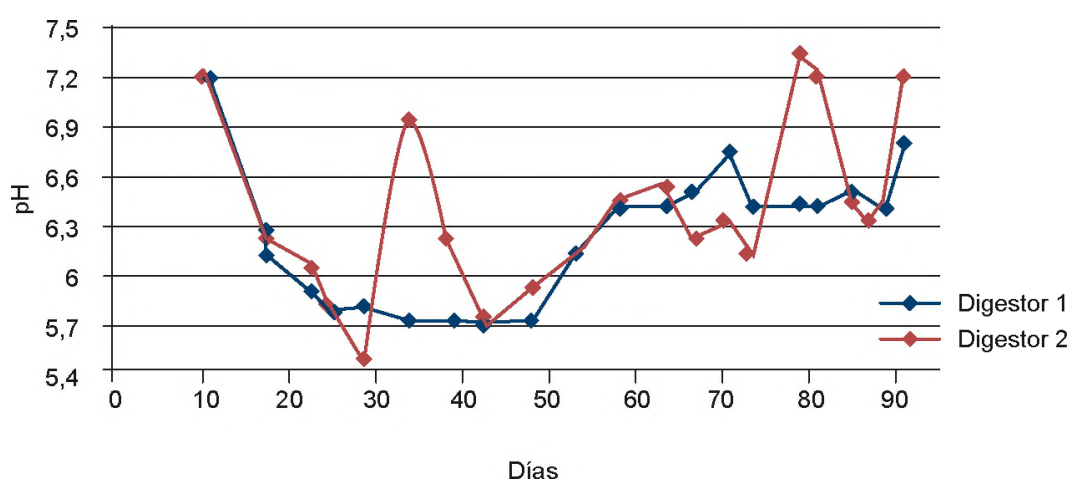

Figura 3. Comportamiento del pH en los digestores.

Es importante tener en cuenta la relación entre el pH y la temperatura. Si las temperaturas están dentro del rango mesofilico y los valores de $\mathrm{pH}$ son ácidos, los microorganismos presentes en los digestores realizan rápidamente su proceso degradativo y generan acidificación; por tanto menor cantidad de gas metano. Por el contrario, si el pH es cercano a la neutralidad los microorganismos actuarán lentamente para degradar la carga orgánica lo que facilita el desarrollo de la etapa metanogénica y produce mayor volumen de biogás.

\section{Tiempo de retención hidráulica}

Como se puede observar en la ecuación 1, se obtuvo un tiempo de retención de 66 días, que es el tiempo en el que las bacterias ya han metabolizado los sólidos volátiles del sustrato y dan paso a la etapa metanogénica del proceso.

\section{Caudal de entrada del efluente}

Para obtener el caudal de entrada del afluente, es necesario conocer el volumen del reactor. En la experimentación realizada se obtuvo un caudal de $0,63 \mathrm{~mL} / \mathrm{min}$, sin embargo, el caudal no fue un parámetro influyente en el proceso ya que el proceso se realizó en una sola etapa y con una única alimentación, es decir, que ese fue el caudal para el momento en el que la mezcla ingresó al digestor.

\section{Volumen de materia orgánica}

Teniendo en cuenta que el volumen total del reactor es de $60 \mathrm{~L}$, el $70 \%$ del volumen del reactor correspondió al volumen de materia orgánica, es decir, $42 \mathrm{~L}$. Considerando que la mezcla en el proceso debe cumplir con la relación (1:3) esto quiere decir que la tercera parte de $42 \mathrm{~L}$ será el volumen de agua que deberá agregarse a la mezcla, es decir, que el volumen necesario de estiércol es 10,5 L y de agua $31,5 \mathrm{~L}$, completando una mezcla de $42 \mathrm{~L}$ que se introdujo al reactor.

\section{Construcción de los digestores}

El proceso de digestión se realizó en tanques de polietileno de alta densidad. En el mercado se encuentran disponibles tanques tanto de polietileno recuperado como original; su diferencia está en el 
tipo de resina de la que están fabricados. Considerando que el polietileno recuperado es un material reciclado, se escogió este tipo de tanque, puesto que cumple con los requerimientos del proceso y el impacto medio ambiental en su fabricación, fue menor. Los tanques son verticales, cualidad que define la forma del digestor y corresponde, a su vez, a la forma establecida para los reactores UASB. El reactor cumple con las siguientes especificaciones:

- Diámetro de la tapa: $60 \mathrm{~cm}$.

- Altura del reactor: $60 \mathrm{~cm}$.

- Ancho: $40 \mathrm{~cm}$

De acuerdo a las ecuaciones $3,4,5$ y 6 , descritas anteriormente se obtuvieron los cálculos para los dos digestores.

$$
\begin{gathered}
A_{R}=\frac{\pi(0.4 \mathrm{~m})^{2}}{4}=0.13 \mathrm{~m}^{2} \\
C_{H}=\frac{6,3 \times 10-7 \mathrm{~m}^{3} / \mathrm{dia}}{0.13 \mathrm{~m}^{2}}=\frac{4.84 \times 10-6 \mathrm{~m}}{\text { dia }} \\
F=0,06 \mathrm{~m}^{3} * 0,042 \mathrm{~m}^{3} / \mathrm{dia} \\
F=\frac{2,52 \times 10-3 \mathrm{kgDQO}}{\mathrm{dia}} \\
V_{R}=4 * \frac{4,84 \times 10-6 \mathrm{~m}}{\text { dia }}=1,936 \times 10-5 \mathrm{~m} / \mathrm{dia}
\end{gathered}
$$

\section{Alternativa para la estabilización de la temperatura interna del nuevo prototipo}

Surgió de la necesidad de controlar el parámetro que afecta directamente el proceso de digestión, evitando en lo posible las variaciones en la temperatura ya mencionadas. Después de realizar una búsqueda bibliográfica (Herrera, 2014), se encontraron las siguientes opciones para realizar la mejora al digestor: recirculación externa de agua caliente, espuma de poliuretano y plancha de corcho. La espuma de poliuretano es el material que tiene mayores ventajas y beneficios para estabilizar la temperatura, por lo cual se realizó un recubrimiento en este material duroplástico. Esta espuma que tiene la ventaja de no ser fusible; presentar un alto punto de ablandamiento y, por tanto, una buena estabilidad térmica, tiene la propiedad de amoldarse a diversas superficies, por lo que pudo adaptarse al contorno del digestor, lo cubrió por completo y aseguró una mejor resistencia térmica (González, 2012).

Adicionalmente, se elaboró un invernadero empleando el plástico de polietileno de alta densidad. 
LÍNEA DE INVESTIGACIÓN: ENERGÍAS LIMPIAS - BIOMASA

\section{Volumen de biogás esperado}

Tal como se verifica en la ecuación 7 el volumen de biogás estimado para el final del proceso en los digestores fue de $0,021 \mathrm{~m} 3$.

$$
V_{\text {Biogás Total }}=1,36 \text { * } 0,27 \text { * } 42 L=20,97 L=0,021 m^{3}
$$

Una vez conocido el volumen de biogás esperado, se buscó un recipiente de volumen similar pero con mayor capacidad.

\section{Selección del tipo de almacenamiento para el biogás}

El almacenamiento del biogás se realizó en dos gasómetros (Figura 4). Sus medidas eran $36 \mathrm{~cm}$ de alto y $30 \mathrm{~cm}$ de diámetro, para un volumen de $0,025 \mathrm{~m}^{3}$.

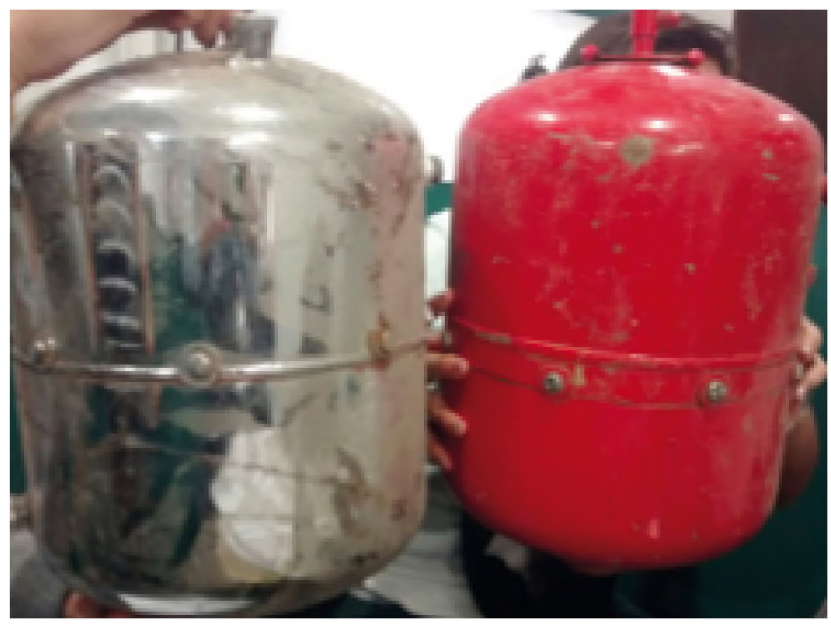

Figura 4. Gasómetros utilizados para almacenar el biogás

\section{Evaluación de la carga microbiológica}

Con el fin de verificar que los microorganismos necesarios estuvieran en la mezcla de los digestores para que ocurriera la degradación de la materia orgánica, se realizó una prueba de coloración de Gram el día 19. Se practicó para el medio de cultivo BHI, el cual no tiene inhibidores y en el que se verificó la existencia de bacterias Gram positivas y Gram negativas (ver Figura 5). También se realizó para carboximetil celulosa y almidón, para comprobar la presencia de microorganismos con enzimas para degradar la celulosa y el almidón y por tanto, asegurar que exista la primera etapa de la digestión, la hidrólisis. 

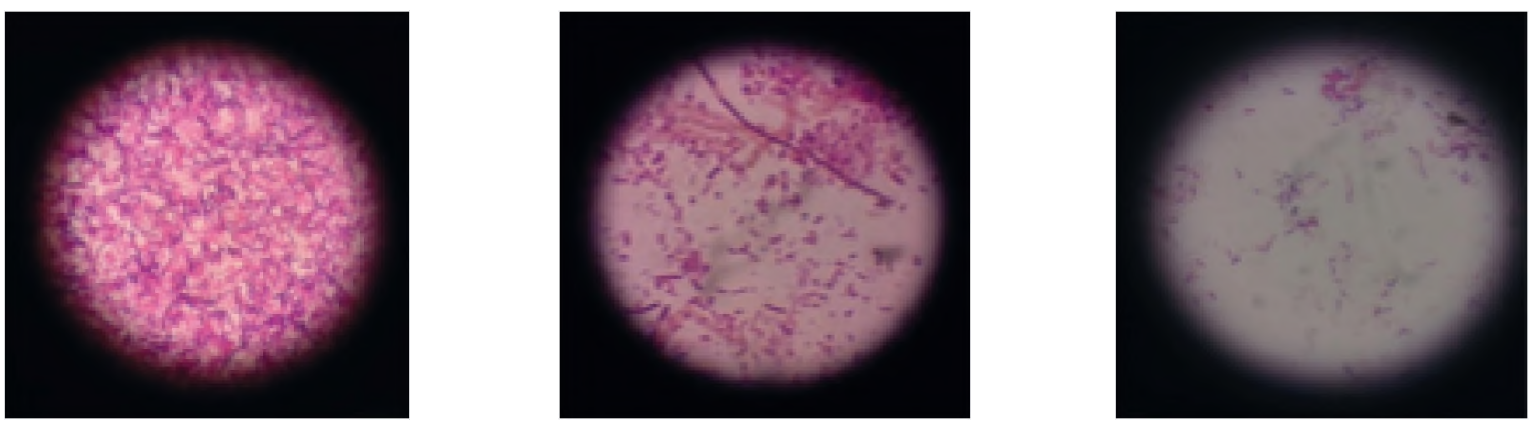

Figura 5. Resultado de la coloración Gram para los cultivos BHI, Carboximetil y almidón respectivamente.

\section{Evaluación de la actividad enzimática de los microorganismos presentes en los digestores}

La actividad enzimática celulolítica resultó positiva: se observó que los microorganismos degradaron el medio; sin embargo, comparado con los medios de almidón y de leche, este medio tuvo una menor proporción. La actividad proteolítica se reflejó en los aros de degradación que se pueden observar en la Figura 6, esto muestra la cantidad de microorganismos que se encuentran en la materia orgánica presente dentro de los reactores, teniendo así la capacidad de degradación para la producción de metano. Al evaluar la actividad amilolítica, se observó que hubo mayor degradación de almidón por bacterias, al igual que de la proteína. En la Figura 6 se observa cómo las bacterias inmersas en la materia orgánica tienen la capacidad de atravesar la fase hidrolítica con mayor rapidez ya que el sustrato contiene mayor cantidad de celulosa, amilasa y proteasas, por lo que los microorganismos presentes podrán degradar correctamente el sustrato.
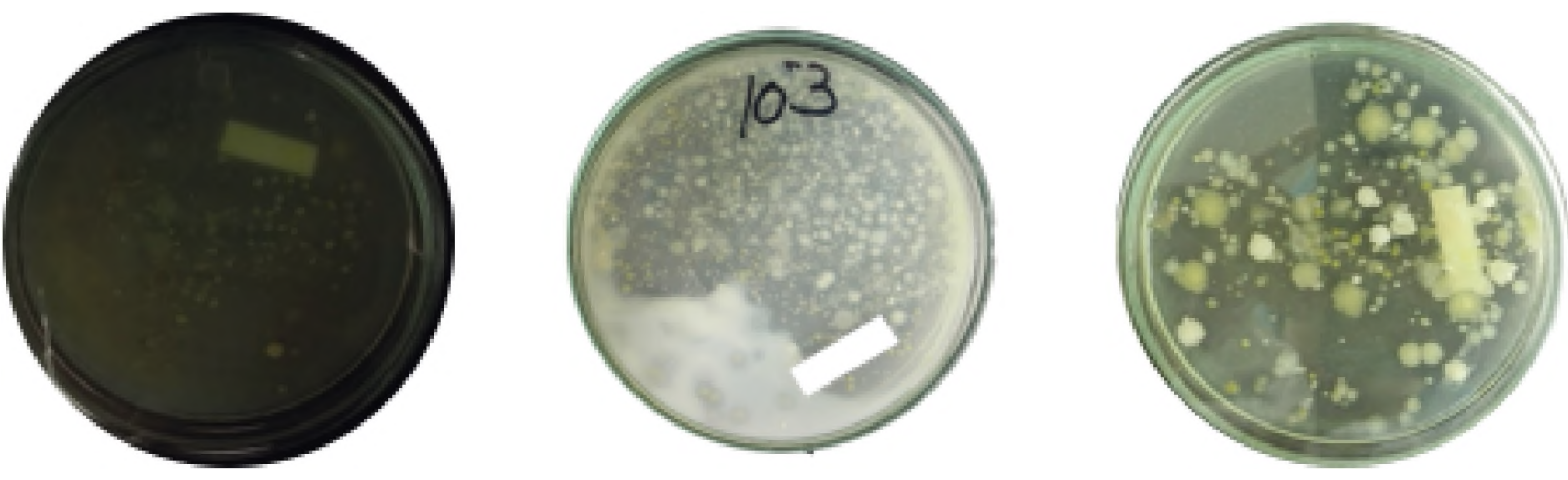

Figura 6. Resultado para los medios de cultivo con celulosa, leche y almidón respectivamente.

Respecto a la lignina, se esperaron 72 horas debido a que es un medio más complejo para ser degradado por los microorganismos. El resultado, como se puede observar en la Figura 7 , fue negativo, es decir, no se presentó degradación en el medio. Esto sucede debido a que los hongos son los mayores degradadores de lignina y en reactores anaerobios no debe haber hongos, lo que asegura de esta manera el buen funcionamiento de los reactores. 


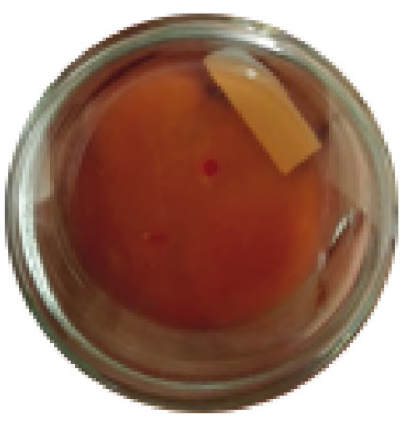

Figura 7. Resultado para el medio de cultivo lignina.

\section{Nutrientes y relación $\mathrm{C} / \mathrm{N}$}

De acuerdo a las pruebas de laboratorio realizados al sustrato inicial, se estableció que el sustrato que ingresó al digestor obtuvo un $65 \%$ de carbono orgánico oxidable, porcentaje significativo para que los microorganismos degraden la materia orgánica.

\section{$D Q O$}

Hay que tener en cuenta que para analizar el comportamiento de la DQO, fue necesario llenar el digestor en su totalidad el día 53 debido a que se identificó un olor no cualitativo de la digestión anaerobia. Al observar el aumento de la DQO (Figura 8), se infiere que agregar un volumen de mezcla adicional ocasiona que el parámetro no disminuya como se espera, debido a que los microorganismos deben acoplarse a las características del medio nuevamente, es decir, que el arranque del proceso se da por segunda vez.

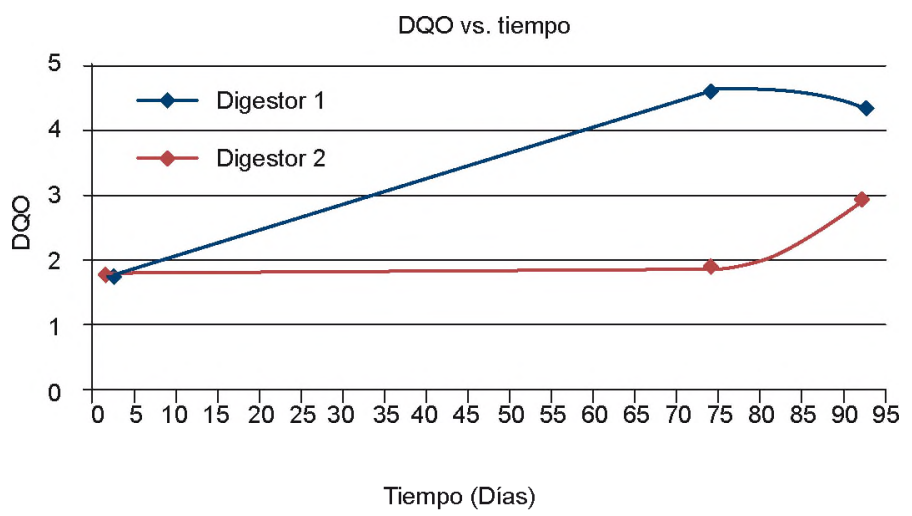

Figura 8. Comportamiento de la DQO en los digestores.

También se puede observar que el sustrato inicial obtuvo el mismo valor de DQO $(1,57)$ en cada digestor; sin embargo, después de realizar el llenado el valor de la DQO aumenta en el digestor 1 en relación al 2, lo que señala mayor cantidad de materia orgánica a oxidar, lo que en consecuencia dificulta la realización de la etapa metanogénica en el digestor 1. En el caso del digestor 2, al obtener valores de DQO menores, es decir, menor cantidad de oxígeno, se facilita la acción metanogénica de los microorganismos. 


\section{Solidos suspendidos, volátiles y totales}

El llenado del día 53 provocó que el porcentaje de solidos totales, volátiles y suspendidos aumentaran dentro de los digestores; no obstante, de acuerdo a los resultados obtenidos durante el proceso, los valores del digestor 2 son menores en comparación al digestor 1, lo que indica mayor degradación dentro del reactor y a su vez generación de biogás en menor tiempo.

\section{Volumen de biogás generado}

El volumen de biogás fue de $0,015 \mathrm{~m}^{3}$ y $0,018 \mathrm{~m}^{3}$ para el digestor 1 y 2 respectivamente en el día 101 del proceso.

\section{Cromatografía de gases}

Como se puede observar en la tabla 2, el porcentaje en volumen de metano para el digestor 2 fue mayor un $9,19 \%$ en relación al 1 , en consecuencia la calidad y cantidad de biogás del digestor 2 corresponde a una mayor eficiencia en el proceso. En cuanto al oxígeno, el digestor 1 fue mayor $24,6 \%$ en relación al 2; es decir, como el proceso trabaja en condiciones anaerobias, el digestor 1 toma mayor tiempo para la producción de biogás, lo cual se verifica con el comportamiento de la DQO.

Tabla 2.

Resultados cromatografía de gases para cada digestor

\begin{tabular}{lcc}
\hline \multirow{2}{*}{ Compuesto } & Digestor 1 & Digestor 2 \\
\cline { 2 - 3 } & \% Volumen & \% Volumen \\
\hline Metano $\left(\mathrm{CH}_{4}\right)$ & 58,3 & 64,2 \\
Oxígeno $\left(\mathrm{O}_{2}\right)$ & 14,2 & 10,7 \\
Hidrógeno $\left(\mathrm{H}_{2}\right)$ & n.d & n.d \\
\hline
\end{tabular}

Respecto al tiempo, el día 101 se obtiene mayor cantidad de biogás en el digestor 2, esto indica que el tiempo de producción de biogás fue menor para el digestor 2 con respecto al digestor 1 .

\section{ANOVA}

Para identificar la influencia de la temperatura, $\mathrm{pH}, \mathrm{DQO}$, y sólidos totales sobre el proceso en el digestor 1 y 2, se realizó un ANOVA por cada parámetro con un diseño balanceado. El análisis se realizó a un nivel de significancia de 0,05 y 0,025 , que dio un $90 \%$ de confiabilidad a los datos arrojados experimentalmente.

De acuerdo a los resultados, la temperatura y $\mathrm{pH}$, son los parámetros que tienen influencia sobre la producción de biogás en el proceso, corroborando de esta manera lo planteado teóricamente. En el caso de DQO y sólidos totales, son parámetros que no incidieron en el proceso, debido a que su comportamiento se vio afectado por un llenado adicional de sustrato que fue realizado posterior al inicio de la experimentación. Al observar los resultados de la distribución $\mathrm{F}$ calculada a los dos niveles de significancia, se asegura que los datos del ANOVA son confiables para cada parámetro. 
LÍNEA DE INVESTIGACIÓN: ENERGÍAS LIMPIAS - BIOMASA

\section{CONCLUSIONES}

Se determinó que para el correcto funcionamiento del proceso de digestión, las condiciones de operación que deben ser estudiadas y controladas son temperatura, $\mathrm{pH}$, tiempo de retención hidráulica, caudal de entrada del afluente y volumen de carga de materia orgánica. Las condiciones más importantes y que representaron una influencia determinante sobre el proceso fueron temperatura y $\mathrm{pH}$.

Los resultados de composición en cuanto a nutrientes, carbono orgánico oxidable y relación C/N para el sustrato inicial fueron significativos para dar lugar a la digestión. Por otra parte, las pruebas microbiológicas permitieron identificar la existencia de microorganismos y aseguraron la degradación de la materia orgánica.

De esta manera y analizando los resultados de la cromatografía de gases realizada al biogás generado en cada digestor, la cantidad de metano obtenida fue de $58,3 \%$ vol y $64,2 \%$ vol para el digestor convencional y nuevo prototipo respectivamente. Esto asegura que la calidad y cantidad en el digestor modificado fue mayor. Es por esto, que la mejora propuesta para estabilizar la temperatura tuvo un efecto positivo y fue determinante para que la producción y calidad del biogás fuera mayor en el nuevo prototipo.

\section{REFERENCIAS}

Galvis Pinzon, Acevedo, D. (2008). Evaluacion del potencial energetico de la biomasa redisual proveniente del sector porcino en colombia2E. Bucaramanga: Universidad industrial de santander Escuela de Ingenieria Quimica.

Hernández, Prada, H. (s.f.). Atlas de potencial energetico de la biomasa residual en Colombia. CoIombia: Upme. Ideam, colciencias, universidad industrial de santander.

Adriana., H. (2014). El estiércol de vaca, reservorio de bacterias resistentes a los antibióticos: El gran temor es que se manifiesten en agentes patógenos responsables de intoxicaciones alimentarias o infecciones hospitalarias. El nacional Caracas Venezuela.

Alvaro, G. J. (2012). Criterios de formulacion de espuma flexible de espuma de poliuretano basados en la evaluacion cualitativa de propiedades finales a nivel laboratorio. Bogota: Universidad Nacional.

Apellido, N. (Año). Capítulo. En Editores, Titulo Libro (Vol. YY). Ciudad: Editorial.

Bautista, A. (2010). Sistema biodigestor para el tratamiento de desechos orgánicos. Leganés: Universidad Carlos III de Madrid. Escuela Politécnica Superior.

Bitton, G. (1994). Wastewater Microbiology. Weley-Liss, New York, USA.

Bohórquez Guitiérrez Lina, L. R. (2004). Estudio de la actividad enzimática celulolítica en la etapa termófila de una fermentación en estado sólido realizada a partir de residuos generados en 
el procesamiento industrial de Papa R-12. Programa de producción agroindustrial. Universidad de la Sabana. Chia.

Borja, S., Martın, T., \& Colmenarejo. (2005). Effect of organic loading rate on the stability, operational parameters and performance of a secondary upflow anaerobic sludge bed reactor treating piggery waste. Bioresource Technology 96.

Chang F., L. C. (2004). Biohydrogen production using an up-flow anaerobic sludge blanket reactor. International journal of hydrogen energy., Page. $33-39$.

Cundinamarca, D. d. (s.f.). Diagnostico situacional del sector.

DANE. (2013). Encuesta Nacional Agropecuaria -ENA- . Bogotá D.C.

Duque, J. B. (2008). Diagnostico del sector porcicola en el municipio de sasaima cundinamarca para determinar el grado de tecnología aplicado en las explotaciones y los problemas más relevantes a los que se enfrenta. Universidad de la salle facultad de administracion de empresas agropecuarias Bogota D.C.

FAO. (2008). Documento técnico de pesca. El uso de hielo en pequeñas embarcaciones de pesca. ISSN 1014-1136, 72.

Guevara Vera, A. (1999). Fundamentos básicos para el diseño de biodigestores anaerobios rurales: Producción de gas y saneamiento de efluentes. Lima: División de salud y ambiente. Centro panamericano de ingenieria sanitaria y ciencias del ambiente.

Hamed M., Z. G., \& Wilko K.P, G. P. (2004). Effect of temperature and temperature fluctuation on thermophilic anaerobic digestion of cattle manure. Faculty of Agriculture, Mansoura University, E1-Mansoura, Egypt. Bioresource Technology 95, 191-20.

Heikkilä, Escobar , G. (s.f.). Biogas production in farms, through anaerobic digestion of cattle and pig manure. Case Studies and research activities in Europe. Finland: TEKES, OPET.

Hogan, K. (1990). Anthropogenic methane emission in the united states. Environmental Enginnering,

Kalpokas. (2011). Experimental investigation on biogas production using pig manure and slaughterhouse waste. Lithuania: Environmental Enginnering, 2029-7092.

León, D. P. (2008). Evaluación del potencial energético de la biomasa residual proveniente del sector porcino en Colombia. Obtenido de Universidad Industrial de Santander : http://repositorio.uis.edu.co/jspui/bitstream/123456789/6765/2/140112.pdf

Lobato. (2012). Estudio de la co-digestión anaerobia de residuos ganadeos con otros substratos agroindustriales. Universidad de León. Instituto de medio ambiente, recursos naturales y biodiversidad. 
LÍNEA DE INVESTIGACIÓN: ENERGÍAS LIMPIAS - BIOMASA

Ministerio de energia. (2011). Manual del biogás. Remoción de barreras para la electrificación rural con energías renovables. Proyecto CHI/00/G32. Chile, 43.

Osorio. S, J. A., Ciro. V, H. J., \& Gonzáles. S, H. (2007). Evaluación de un sistema de biodigestión en serie para clima frio. Universidad Nacional de Colombia. En: Revista Facultad Nacional de Agronomía. Medellín. Vol. 60. ISSN 0304-2847. Medellín.

Pérez Elvira, N. P. ( 2006). Sludge minimisation technologies. Environ. Sci. Biotechnol. 5(4)., 375 398.

Sánchez E, B. R., Travieso L, M. A., \& Colmenarejo. (2005). Effect of organic loading rate on the stability, operational parameters and performance of a secondary upflow anaerobic sludge bed reactor treating piggery waste. Bioresource Technology 96, 335 - 344.

Tamrat, A., Mebeaselassie, A., \& Amare., G. (2013). Co-digestion of cattle manure with organic kitchen waste to increase biogas production using rumen fluid as inoculums. International Journal of Physical Sciences. . Vol.8 (11), Page. 443-450.

UPME. (2007). Informe de gestión upme 2007. Ministerio de Minas y Energía.

UPME. (2010). Atlas del potencial energético de la biomasa residual en Colombia. 31. Bogotá, Colombia. Obtenido de Atlas del potencial energético de la biomasa residual en Colombia: http://www1.upme.gov.co/sites/default/files/article/1768/files/Atlas\%20de\%20Biomasa\%20 Residual\%20Colombia .pdf

UPME. (Marzo 2003). Formulación de un programa básico de normalización para aplicaciones de energías alternativas y difusión. Documento ANC-0603-19-01.

Upton. (1997). Intensification or extensification: which has the lowest environmental burden. WARI RMZ. No 88. FAO Roma. Italia.

Varnero Moreno, M. T. (2011). Manual del Biogás. Santiago de Chile: Gobierno de Chile Ministerio de Energía.

Yank L, M. (2005). Estudio de la producción de biogás en función de la cantidad de residuos de madera en un biodigestor tipo de carga única o batch. Grupo de investigación en Energias Renovables. Universidad Nacional de Nordeste. Argentina, 2. 